\title{
SCIENTIFIC REPORTS

\section{Adherence to nine-month isoniazid for latent tuberculosis infection in healthcare workers: a prospective study in a tertiary hospital}

Sung Jun Chung $\mathbb{1}^{1,2}$, Hyun Lee ${ }^{1,2}$, Gun Woo Koo ${ }^{1}$, Ji-Hee Min ${ }^{1}$, YoomiYeo ${ }^{1}$, Dong Won Park ${ }^{1}$, Tai Sun Park ${ }^{1}$, Ji-Yong Moon ${ }^{1}$, Sang-Heon Kim ${ }^{1 *}$, Tae Hyung Kim ${ }^{1}$, Jang Won Sohn ${ }^{1}{ }^{1}$ \& Ho Joo Yoon ${ }^{1}$

Poor adherence to medication can lead to treatment failure in healthcare workers (HWCs) with latent tuberculosis infection (LTBI) who are at high risk of developing active tuberculosis. However, the factors associated with non-completion of nine-month LTBI treatment with isoniazid $(9 \mathrm{H})$ have not been well studied. We investigated the completion rate and factors affecting adherence to LTBI treatment with $9 \mathrm{H}$ among $\mathrm{HCWs}$. A prospective cohort study of $114 \mathrm{HCWs}$ who were diagnosed with LTBI by QuantiFERON-TB Gold In-Tube tests were performed in a single university hospital between June 2016 and December 2017. All patients received the $9 \mathrm{H}$ LTBI treatment. At each visit, treatment adherence and development of adverse reactions to isoniazid were evaluated via a standard questionnaire. To evaluate the impact of the severity of hepatotoxicity on non-completion of LTBI treatment, we classified hepatotoxicity into two groups: severe hepatotoxicity was defined as alanine aminotransferase $>3.0$ times the upper normal limit (UNL) with symptoms or $=5.0$ times the UNL. Mild hepatotoxicity was defined as alanine aminotransferase $>\mathrm{UNL}$, but not meet the definition of severe hepatotoxicity. Overall, $71 \mathrm{HCWs}(62.3 \%)$ completed LTBI treatment with $9 \mathrm{H}$ while $43 \mathrm{HCWs}(37.7 \%)$ discontinued their treatment. Most discontinuation $(81.4 \%, 35 / 43)$ occurred during the first three months of treatment. There were no significant differences in age, sex, occupation, or comorbidities between the HCWs who completed and those who discontinued LTBI treatment. However, HCWs who discontinued LTBI treatment had more hepatotoxicity than those who completed treatment (44.2\% vs. $11.3 \%, P<0.001)$. Cox proportional hazard analysis revealed that hepatotoxicity is the only factor significantly associated with discontinuation of $9 \mathrm{H} \mathrm{LTBI}$ treatment (unadjusted HR $=2.89,95 \%$ $\mathrm{Cl}=1.62-5.46$ ). In multivariable analysis, not only severe hepatotoxicity (adjusted $\mathrm{HR}=7.99,95 \%$ $\mathrm{Cl}=3.05-20.94)$ but also mild hepatotoxicity was significantly associated with discontinuation of LTBI treatment (adjusted $\mathrm{HR}=2.34,95 \% \mathrm{Cl}=1.05-5.21$ ). The completion rate of $9 \mathrm{H} \mathrm{LTBI}$ treatment was $62.3 \%$ among HCWs. While age, sex, occupation, and pretreatment comorbidities were not associated with treatment completion, isoniazid-induced hepatotoxicity significantly affected adherence.

Healthcare workers (HCWs) are vulnerable to Mycobacterium tuberculosis infection due to a higher risk of exposure to patients with active pulmonary tuberculosis ${ }^{1-3}$. Latent tuberculosis infection (LTBI) among HCWs has been reported to be as high as $64 \%$, worldwide ${ }^{4,5}$. Three studies in Korea reported that LTBI prevalence was about $15 \%-17 \%$ among HCWs ${ }^{3,6,7}$. In our previous study, about one-third of HCWs were diagnosed with LTBI by interferon-gamma releasing assay (IGRA), which was higher than reported by other studies ${ }^{8}$.

Successfully treating HCWs for LTBI is crucial for tuberculosis (TB)-control because HCWs who develop active pulmonary TB can easily transmit their infections to patients. Across communities, the adherence rate for LTBI treatment is unsatisfactory, ranging from $44 \%-81 \%$ in the general population ${ }^{9-15}$ and $17 \%-100 \%$ among HCWs $^{7,12,14,16-19}$. However, studies that evaluated LTBI adherence rates among HCWs were limited by their 


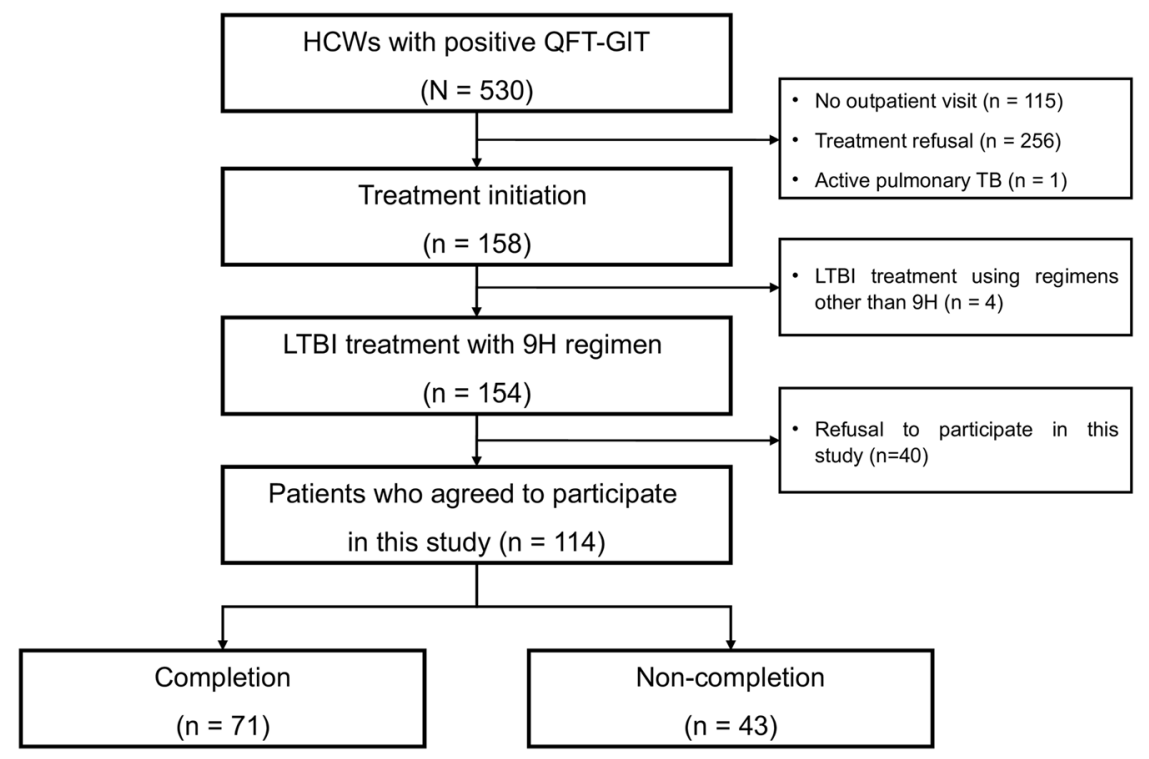

Figure 1. Flow chart of LTBI treatment in HCWs who were diagnosed with LTBI. LTBI latent tuberculosis infection, HCW healthcare worker, QFT-GIT QuantiFERON-TB Gold In-Tube tests, OPD outpatient department, $9 H$ 9-month treatment with isoniazid.

retrospective study designs, variable treatment regimens, and relatively small subject populations, all of which could explain the wide range of completion rates observed.

The currently recommended LTBI treatment regimens according to the American Thoracic Society (ATS), the Centers for Disease Control and Prevention (CDC), Infectious Diseases Society of America (IDSA), and the World Health Organization (WHO) include nine-month treatment with isoniazid $(9 \mathrm{H})$, four-month treatment with rifampin (4R), or three-month treatment with a combination of isoniazid and rifampin (3HR), with a broad preference for the $9 \mathrm{H}$ regimen ${ }^{20,21}$. The Korean guidelines for LTBI treatment also adopted these recommendations $^{22}$. Accordingly, the $9 \mathrm{H}$ regimen is widely used in clinical practice as the preferred treatment. However, given that it is the longest treatment regimen, there is concern that it has an inferior completion rate compared with other regimens ${ }^{13}$. However, few studies have prospectively evaluated this concern among HCWs.

Thus, we prospectively evaluated the completion rate and factors associated with adherence to the $9 \mathrm{H}$ regimen among HCWs with LTBI who were diagnosed by IGRA.

\section{Methods}

Study design and population. A prospective cohort study to assess completion rate and factors affecting adherence to LTBI treatment among HCWs was performed in a single university hospital between June 2016 and December 2017. During the study period, QuantiFERON-TB Gold In-Tube tests (QFT-GIT; Cellestis Ltd., Carnegie, VIC, Australia) were performed for 2,379 HCWs; 530 HCWs were positive according to QFT-GIT and $158 \mathrm{HCW}$ visited an outpatient clinic and agreed to begin LTBI treatment. After excluding four HCWs who received a treatment regimen other than $9 \mathrm{H}$ and $40 \mathrm{HCWs}$ who refused to participate in this study, a total of 114 HCWs participated in this study (Fig. 1). Data on 86 patients who agreed to participate in this study were previously reported ${ }^{8}$.

Ethics approval and consent to participate. Written informed consent was obtained from each HCW who participated in this study. The study protocol was approved by the Institutional Review Board of Hanyang University Hospital (IRB number 2017-02-018). All methods were performed in accordance with the relevant guidelines and regulations.

LTBI treatment protocol, follow-up, and monitoring of adverse reactions. All HCWs who agreed to LTBI treatment received isoniazid at a dose of $5 \mathrm{mg} / \mathrm{kg}$ up to $300 \mathrm{mg}$ once daily for nine months (270 doses) ${ }^{21}$. Generally, participants were followed-up at two-week intervals for one month, followed by one-month intervals until treatment completion. At each visit, participants were asked to report any adverse events via a structured form. All suspected adverse reactions were investigated, managed, and reported according to standardized in-hospital protocol. During the $9 \mathrm{H}$ treatment, patients reported all their remaining drug doses to every visit for pill counts.

Using a standard questionnaire, brief physical assessment, and routine laboratory examination, patients were evaluated to check for the following adverse events: gastrointestinal symptoms, dermatologic symptoms and signs, myelosuppression, hepatotoxicity, peripheral neuropathy, and other symptoms. To evaluate the impact of the severity of hepatotoxicity on non-completion of LTBI treatment, we classified hepatotoxicity into two groups: severe hepatotoxicity was defined as alanine aminotransferase $>3.0$ times the upper normal limit (UNL) with symptoms or $>5.0$ times the UNL, according to the American Thoracic Society guideline ${ }^{23}$ 


\begin{tabular}{|l|l|}
\hline & Total $(\mathbf{N}=1 \mathbf{1 1})$ \\
\hline Age, years & $42(32-52)$ \\
\hline Sex, female & $79(69.3)$ \\
\hline BMI, $\mathrm{Kg} / \mathrm{m}^{2}$ & $23.1(19.6-26.6)$ \\
\hline Occupation & \\
\hline Patient-related HCWs & $89(78.1)$ \\
\hline Physicians & $11 / 89(12.4)$ \\
\hline Nurses & $54 / 89(60.7)$ \\
\hline Others & $24 / 89(26.9)$ \\
\hline Patient-unrelated HCWs & $25(21.9)$ \\
\hline Comorbidity & \\
\hline Hypertension & $10(8.8)$ \\
\hline Chronic liver disease & $4(3.5)$ \\
\hline Diabetes mellitus & $2(1.8)$ \\
\hline Chronic kidney disease & $1(0.9)$ \\
\hline
\end{tabular}

Table 1. Baseline characteristics of the study population. Data are presented as either medians and interquartile ranges or as numbers and percentages, as appropriate. ${ }^{\dagger}$ Patient-unrelated HCWs included administrative staff, cooks, and cleaning staff. $B M I$ body mass index, $H C W$ healthcare worker.

and Korean guideline ${ }^{22}$. Mild hepatotoxicity was defined as alanine aminotransferase $>$ UNL, but not meet the definition of severe hepatotoxicity. Gastrointestinal symptoms included nausea, anorexia, vomiting, and diarrhea. Dermatological symptoms and signs included itching, urticaria, or rash. Myelosuppression was defined as neutropenia (absolute neutrophil count $<1,000 / \mathrm{mm}^{3}$ ), thrombocytopenia (platelet $<100,000 / \mathrm{mm}^{3}$ ), or anemia $(\mathrm{Hb} \leq 10.5 \mathrm{~g} / \mathrm{dL})$. Other symptoms included headache, dizziness, general weakness, fatigue, or fever.

Outcomes. The primary outcomes of interest were completion rates and factors associated with non-completion among HCWs who received the $9 \mathrm{H}$ regimen for LTBI treatment. Treatment completion was defined as receipt of $\geq 80 \%$ of isoniazid doses within nine months of treatment initiation ${ }^{13,15}$.

Statistical analyses. Categorical variables are presented as numbers with percentages, and continuous variables are presented as medians with interquartile ranges (IQR) or means with standard deviations, as appropriate. Pearson's chi-square test or Fisher's exact test were used to comparing categorical variables. Student's t-test or the Mann-Whitney U test was used to compare continuous variables. We used the Kaplan-Meier method to estimate the proportion of HCWs who discontinued LTBI treatment during follow-up.

To evaluate the factors affecting LTBI treatment adherence, univariable and multivariable Cox proportional hazard analysis was performed. Factors included in the Cox models included age, sex, body mass index, hypertension, chronic liver disease, work group (patient-related HCWs vs. patient-unrelated HCWs), hepatotoxicity, gastrointestinal symptoms, dermatologic symptoms, hematologic symptoms, peripheral neuropathy, and other adverse reactions. All tests were two-tailed, and a $P$-value $<0.05$ was considered statistically significant. All statistical analyses were performed using the Statistical Package for the Social Sciences (SPSS) for Windows (version 24.0; IBM Corp., Armonk, NY, USA) and STATA (version 15.0; Stata Corporation, College Station, TX, USA).

Results

Study subjects. The baseline characteristics of the 114 subjects who initiated $9 \mathrm{H}$ treatment are summarized in Table 1 . The median age was 42 years, and $69.3 \%(n=79)$ were female. The median body mass index was $23.1 \mathrm{~kg} / \mathrm{m}^{2}$. Subjects included 89 patient-related HCWs $(78.1 \%)$ and 25 patient-unrelated HCWs (21.9\%). The common comorbidities were hypertension $(n=10,8.8 \%)$, chronic liver disease $(n=4,3.5 \%)$, diabetes mellitus $(\mathrm{n}=2,1.8 \%)$, and chronic kidney disease $(\mathrm{n}=1,0.9 \%)$. None of the HCWs tested positive for human immunodeficiency virus infection.

Completion rate. As shown in Table 2, while 62.3\% of HCWs (71/114) completed LTBI treatment, 37.7\% (43/114) discontinued LTBI treatment. The completion proportions were 54.5\% (12/22) in the twenties, $56.3 \%$ $(9 / 16)$ in the thirties, $66.7 \%(34 / 51)$ in the forties, and $64.0 \%(16 / 25)$ in the fifties, respectively. By sex, the completion proportions were $62.9 \%$ among men and $62.0 \%$ among women. The proportions of completion by occupation were 54.5\% (6/11) among doctors, 63.0\% (34/54) among nurses, 62.5\% (15/24) among other patient-related HCWs, and $64.0 \%(16 / 25)$ among patient-unrelated HCWs.

As shown in Fig. 2, during the first three months of treatment duration, 35 patients $(30.7 \%)$ discontinued treatment, and a total of 41 patients $(36.0 \%)$ discontinued treatment by month six.

Factors affecting non-completion. As shown in Table 3, the overall adverse reaction rate was $61.4 \%$ (70/114), which includes $57.7 \%$ of HCWs who completed and $67.4 \%$ of HCWs who discontinued LTBI treatment $(P=0.422)$. As shown in Table 3, there were no significant differences in the rates of adverse reactions, including gastrointestinal $(38.0 \%$ vs. $27.9 \%$; $P=0.350)$, dermatologic $(11.3 \%$ vs. $16.3 \%$; $P=0.129)$, hematologic $(18.3 \%$ vs. $18.6 \% ; P=0.250)$, peripheral neuropathy $(2.8 \%$ vs. $9.3 \% ; P=0.133)$, and others $(36.6 \%$ vs. $30.2 \% ; P=0.523)$, 


\begin{tabular}{|l|l|l|l|}
\hline & $\begin{array}{l}\text { Total } \\
(\mathbf{N}=114)\end{array}$ & $\begin{array}{l}\text { Completion } \\
(\mathbf{n = 7 1 )}\end{array}$ & $\begin{array}{l}\text { Non-completion } \\
(\mathbf{n = 4 3 )}\end{array}$ \\
\hline Age, years & $44(34-49)$ & $44(34-54)$ & $43(33-53)$ \\
\hline Twenties & $22(19.3)$ & $12 / 22(54.5)$ & $10 / 22(45.5)$ \\
\hline Thirties & $16(14.0)$ & $9 / 16(56.3)$ & $7 / 16(43.7)$ \\
\hline Forties & $51(44.7)$ & $34 / 5(66.7)$ & $17 / 51(33.3)$ \\
\hline Fifties & $25(21.9)$ & $16 / 25(64.0)$ & $9 / 25(36.0)$ \\
\hline Sex & & & \\
\hline Male & $35(30.7)$ & $22 / 35(62.9)$ & $13 / 35(37.1)$ \\
\hline Female & $79(69.3)$ & $49 / 79(62.0)$ & $30 / 79(38.0)$ \\
\hline Occupation & & & \\
\hline Patient-related HCWs & $89(78.1)$ & $55 / 89(61.8)$ & $34 / 89(38.2)$ \\
\hline Doctors & $11(15.5)$ & $6 / 11(54.5)$ & $5 / 11(45.5)$ \\
\hline Nurses & $54(47.4)$ & $34 / 54(63.0)$ & $20 / 54(37.0)$ \\
\hline Others & $24(21.1)$ & $15 / 24(62.5)$ & $9 / 24(37.5)$ \\
\hline Patient-unrelated HCWs & $25(21.9)$ & $16 / 25(64.0)$ & $9 / 25(36.0)$ \\
\hline Comorbidity & & & \\
\hline Hypertension & $10(8.8)$ & $8 / 10(80.0)$ & $2 / 10(20)$ \\
\hline Chronic liver disease & $4(3.5)$ & $2 / 4(50.0)$ & $2 / 4(50)$ \\
\hline Diabetes mellitus & $2(1.8)$ & $2 / 2(100.0)$ & $0 / 2(0)$ \\
\hline Chronic kidney disease & $1(0.9)$ & $1 / 1(100.0)$ & $0 / 1(0)$ \\
\hline
\end{tabular}

Table 2. Comparison of the clinical characteristics of HCWs who completed versus those who discontinued 9-month isoniazid treatment. Data are presented as either medians and interquartile ranges or as numbers and percentages, as appropriate. ${ }^{*}$ Others included radiology technicians, laboratory technicians, physical therapists, and medical technicians. ${ }^{\dagger}$ Patient-unrelated HCWs included administrative staff, cooks, and cleaning staff. $H C W$ healthcare worker, $L T B I$ latent tuberculosis infection.

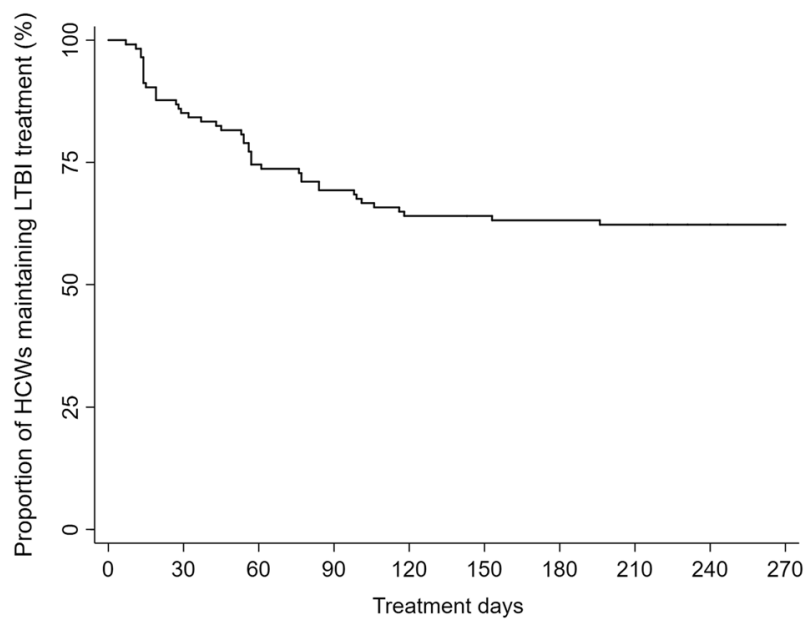

Figure 2. The proportion of HCWs on LTBI treatment with $9 \mathrm{H}$ over the study period. HCW healthcare worker, $9 \mathrm{H}$, 9-month treatment with isoniazid.

between HCWs who completed and those who discontinued LTBI treatment. In contrast, hepatotoxicity was significantly higher among HCWs who discontinued compared with those who completed LTBI treatment $(44.2 \%$ vs. $11.3 \%, P<0.001)$. Of the 27 HCWs who had hepatotoxicity, $19(70.4 \%)$ discontinued LTBI treatment, about two-thirds of whom developed hepatotoxicity within three months of LTBI treatment initiation. None of the HCWs died from adverse reactions.

Next, to determine significant factors contributing to non-completion of LTBI treatment, univariable and multivariable Cox proportional regression analyses were performed. Hepatotoxicity was the only clinical factor that significantly affected the non-completion of LTBI among HCWs who initiated LTBI treatment. Compared with HCWs without hepatotoxicity, those with mild hepatotoxicity and severe hepatotoxicity were 2.34 (95\% CI, 1.05-5.21) and 7.99 (95\% CI, 3.05-20.94) times more likely to discontinue LTBI treatment, respectively (Table 4). 


\begin{tabular}{|l|l|l|l|l|}
\hline & $\begin{array}{l}\text { Total } \\
(\mathbf{N}=114)\end{array}$ & $\begin{array}{l}\text { Completion } \\
(\mathbf{n}=7 \mathbf{7})\end{array}$ & $\begin{array}{l}\text { Non-completion } \\
(\mathbf{n}=\mathbf{4 3})\end{array}$ & $\boldsymbol{P}$ \\
\hline Any adverse reactions & $70(61.4)$ & $41(57.7)$ & $29(67.4)$ & 0.422 \\
\hline Gastrointestinal & $39(34.2)$ & $27(38.0)$ & $12(27.9)$ & 0.350 \\
\hline Nausea & $27(23.7)$ & $16(22.5)$ & $11(25.6)$ & \\
\hline Anorexia & $8(7.0)$ & $6(8.5)$ & $2(4.7)$ & \\
\hline Diarrhea & $2(1.8)$ & $2(2.8)$ & $0(0)$ & \\
\hline Skin & $15(13.2)$ & $8(11.3)$ & $7(16.3)$ & 0.129 \\
\hline Itching & $12(10.5)$ & $6(8.5)$ & $6(14.0)$ & \\
\hline Urticaria & $4(3.5)$ & $1(1.4)$ & $3(7.0)$ & \\
\hline Eczema & $4(3.5)$ & $3(4.2)$ & $1(2.3)$ & \\
\hline Hematologic & $21(18.4)$ & $13(18.3)$ & $8(18.6)$ & 0.250 \\
\hline Neutropenia & $6(5.3)$ & $3(4.2)$ & $3(7.0)$ & \\
\hline Anemia & $12(10.5)$ & $7(9.9)$ & $5(11.6)$ & \\
\hline Thrombocytopenia & $4(3.5)$ & $3(4.2)$ & $1(2.3)$ & \\
\hline Hepatotoxicity & $27(23.7)$ & $8(11.3)$ & $19(44.2)$ & $<0.001$ \\
\hline Mild hepatotoxicity & $17(14.9)$ & $7(9.9)$ & $10(23.3)$ & \\
\hline Severe hepatotoxicity & $10(8.8)$ & $1(1.4)$ & $9(20.9)$ & \\
\hline Peripheral neuropathy & $6(5.3)$ & $2(2.8)$ & $4(9.3)$ & 0.133 \\
\hline Others & $39(34.2)$ & $26(36.6)$ & $13(30.2)$ & 0.523 \\
\hline Headache & $10(8.8)$ & $7(9.9)$ & $3(7.0)$ & \\
\hline Dizziness & $12(10.5)$ & $7(9.9)$ & $5(11.6)$ & \\
\hline General weakness & $31(27.2)$ & $21(29.6)$ & $10(23.3)$ & \\
\hline Fever & $4(3.5)$ & $2(2.8)$ & $2(4.7)$ & \\
\hline
\end{tabular}

Table 3. Adverse reactions to LTBI treatment with 9-month treatment with isoniazid. The data are presented as numbers and percentages. * Mild hepatotoxicity was defined as ALT > UNL, but not meet the definition of severe hepatotoxicity. Severe hepatotoxicity was defined as ALT $>3.0$ times UNL with symptoms or $>5.0$ times UNL without symptoms. $A L T$ alanine aminotransferase, $U N L$ upper normal limit.

\section{Discussion}

To the best of our knowledge, this is the first prospective observational study to evaluate completion rates and factors associated with poor adherence to the $9 \mathrm{H}$ LTBI treatment among HCWs. We found that about two-thirds of HCWs who initiated LTBI treatment completed LTBI treatment and one-third discontinued LTBI treatment. Most discontinuation occurred within three months of LTBI treatment initiation, and hepatotoxicity was the only significant factor associated with non-completion of LTBI treatment. We further showed even mild hepatotoxicity, not meeting the definition of hepatotoxicity of the current guideline, was also associated with discontinuation of LTBI treatment.

The completion rate for the $9 \mathrm{H}$ LTBI treatment among HCWs in this study was $62.3 \%$, which is higher than that reported in a previous study $(58 \%)^{19}$. Regarding completion rates for other LTBI treatment regimens among HCWs, approximately $70 \%$ and $80 \%$ of adherence rates were reported with $4 \mathrm{R}^{16,19}, 75 \%$ and $82 \%$ with a six-month isoniazid regimen ${ }^{12,18}$, and $61.7 \%$ and $100 \%$ with $3 \mathrm{HR}^{7,16}$. These results indicate that completion rates for the $9 \mathrm{H}$ regimen among HCWs is relatively lower than those for shorter treatment regimens. However, our study results also show that approximately $80 \%$ of HCWs who did not complete LTBI treatment discontinued within three months of their initial treatment, suggesting that the completion rate would not have been better with a shorter regimen (e.g., four-six-month regimen) and, thus, management of modifiable factors is especially important for improving the LTBI treatment completion rate. In addition, no randomized controlled trials that compare completion rates for the $9 \mathrm{H}$ regimen with shorter regimens have been conducted in HCWs. Thus, a well-designed randomized controlled trial is needed.

In our study, the overall rate of adverse reactions was $61 \%$, and hepatotoxicity occurred in $24 \%$ of HCWs received the $9 \mathrm{H}$ regimen. A previous study found that about $41 \%$ of $\mathrm{HCWs}$ who received the $9 \mathrm{H}$ regimen experienced adverse reactions, and $28 \%$ had hepatotoxicity ${ }^{19}$. The reasons for the relatively lower prevalence of hepatotoxicity despite higher overall adverse reactions in our study relative to the previous study are unclear. The prospective design of our study might be associated with higher adverse reactions because we assessed adverse reactions at one-month intervals. Thus, it is likely that we recorded more mild adverse events, especially considering that most patients, except for those experiencing hepatotoxicity, did not discontinue due to adverse reactions. However, hepatotoxicity prevalence in our study and the previous study might not be directly comparable because the other study did not clearly define their diagnostic criteria for hepatotoxicity. Regarding adverse reactions associated with other regimens, $42 \%$ and $49 \%$ of HCWs experienced adverse reactions to $4 \mathrm{R}^{16,19}, 47 \%$ to $3 \mathrm{HR}^{16}$, and $73 \%$ to a combination of rifapentine and isoniazid ${ }^{19}$. However, because the definitions for adverse events might vary between studies, further studies comparing adverse reactions to various LTBI treatment regimens in HCWs are needed.

Herein, we found that hepatotoxicity was the most significant factor associated with non-completion of LTBI treatment. Of the patients who discontinued LTBI treatment, about $23 \%$ and $20 \%$ developed mild and severe 


\begin{tabular}{|c|c|c|c|c|}
\hline & \multicolumn{2}{|c|}{ Univariable analysis } & \multicolumn{2}{|c|}{ Multivariable analysis ${ }^{\ddagger}$} \\
\hline & $\begin{array}{l}\text { Hazard ratio } \\
(95 \% \text { CI })\end{array}$ & $P$ & $\begin{array}{l}\text { Hazard ratio } \\
(95 \% \mathrm{CI})\end{array}$ & $P$ \\
\hline Age group & $0.99(0.96-1.01)$ & 0.314 & & \\
\hline The twenties & Reference & & Reference & \\
\hline Thirties & $0.93(0.35-2.45)$ & 0.890 & $0.99(0.32-3.10)$ & 0.983 \\
\hline Forties & $0.73(0.34-1.59)$ & 0.432 & $0.70(0.27-1.87)$ & 0.480 \\
\hline Fifties & $0.67(0.26-1.70)$ & 0.401 & $0.43(0.16-1.22)$ & 0.113 \\
\hline Sex, male & $1.06(0.55-2.03)$ & 0.863 & $1.08(0.48-2.44)$ & 0.856 \\
\hline BMI, $\mathrm{kg} / \mathrm{m}^{2}$ & $0.99(0.90-1.08)$ & 0.783 & $1.01(0.91-1.12)$ & 0.873 \\
\hline \multicolumn{5}{|l|}{ Work group } \\
\hline Patient-related HCWs & Reference & & Reference & \\
\hline Patient-unrelated HCWs & $1.13(0.54-2.36)$ & 0.742 & $0.68(0.28-1.63)$ & 0.384 \\
\hline \multicolumn{5}{|l|}{ Comorbidity } \\
\hline Hypertension & $2.02(0.49-8.36)$ & 0.331 & $0.78(0.17-3.55)$ & 0.746 \\
\hline Chronic liver disease & $0.78(0.19-3.21)$ & 0.726 & $0.79(0.13-4.94)$ & 0.803 \\
\hline Adverse reactions & $1.11(0.50-2.49)$ & 0.860 & - & \\
\hline Hepatotoxicity $^{*}$ & $2.89(1.62-5.46)$ & $<0.001$ & - & \\
\hline No hepatotoxicity & Reference & & Reference & \\
\hline Mild hepatotoxicity & $2.26(1.08-4.74)$ & 0.030 & $2.34(1.05-5.21)$ & 0.038 \\
\hline Severe hepatotoxicity & $4.71(2.14-10.34)$ & $<0.001$ & $7.99(3.05-20.94)$ & $<0.001$ \\
\hline Gastrointestinal & $0.85(0.45-1.60)$ & 0.607 & $0.77(0.37-1.62)$ & 0.491 \\
\hline Dermatologic & $1.52(0.73-3.16)$ & 0.266 & $2.25(0.91-5.55)$ & 0.080 \\
\hline Hematologic & $0.64(0.25-1.62)$ & 0.344 & $0.69(0.26-1.85)$ & 0.463 \\
\hline Peripheral neuropathy & $2.24(0.80-6.27)$ & 0.125 & $3.23(0.83-12.51)$ & 0.090 \\
\hline Others $^{\dagger}$ & $0.90(0.48-1.67)$ & 0.738 & $0.98(0.51-1.88)$ & 0.945 \\
\hline
\end{tabular}

Table 4. Cox proportional hazard analysis for factors affecting non-completion during LTBI treatment among HCWs. Data are presented as ratios and 95\% CIs. *Mild hepatotoxicity was defined as ALT > UNL, but not meet the definition of severe hepatoxicity. Severe hepatotoxicity was defined as ALT > 3.0 times UNL with symptoms or $>5.0$ times UNL without symptoms. ${ }^{\dagger}$ Others include headache, dizziness, general weakness, fatigue, and fever. ${ }^{\ddagger}$ Adjusted for age group, sex, BMI, work group, comorbidity (hypertension and chronic liver disease), and each type of adverse events (the severity of hepatotoxicity, gastrointestinal, dermatologic, and hematologic events, peripheral neuropathy, and others). $C I$ confidence interval, $B M I$ body mass index, $H C W$ healthcare worker, UNL upper normal limit.

hepatotoxicity, respectively. When we analyzed discontinuation rate among the patients with hepatotoxicity, surprisingly, not only those with severe hepatotoxicity in whom $90 \%$ discontinued LTBI treatment but also about $60 \%$ of those with mild hepatotoxicity discontinued LTBI treatment. The reasons for this phenomenon are not clear. It seems that the threshold for discontinuation of LTBI treatment due to hepatotoxicity might be lower than that of active pulmonary TB treatment, as the purpose of LTBI treatment is not to treat active disease but to prevent future disease. From this view, our study has provided valuable information that even mild hepatotoxicity can be a barrier to successful LTBI treatment. Accordingly, our results suggest that augmented early management of LTBI treatment adherence with a specific focus on hepatotoxicity management is very important.

This study had several limitations. First, our study was conducted in a single hospital. Because many factors can affect treatment adherence among HCWs, including LTBI prevalence, the occurrence of adverse reactions, and variation in associated stigmas, can affect the adherence of LTBI treatment in different HCWs, our findings might not be generalizable to other hospitals or countries. Second, only a minority of HCWs diagnosed with LTBI presented for treatment. Therefore, our study might have overestimated the completion rate by pre-selecting individuals inclined to seek treatment. Third, as we did not evaluate the reasons for follow-up loss, we could not identify specific reasons for discontinuation of LTBI treatment in these HCWs.

\section{Conclusions}

In conclusion, in our study, the completion rate was $62.3 \%$ with LTBI treatment with $9 \mathrm{H}$ among HCWs. Most HCWs discontinued LTBI during the first 3months after treatment initiation. Of the various adverse events, hepatotoxicity was the only significant factor associated with discontinuation. The strategies to enhance adherence during the early phage of LTBI treatment and managing hepatotoxicity properly are needed for the successful management of LTBI treatment among HCWs who receive $9 \mathrm{H}$.

\section{Data availability}

The data and analytic methods are available upon request.

Received: 29 October 2019; Accepted: 19 March 2020;

Published online: 15 April 2020 


\section{References}

1. Baussano, I. et al. Tuberculosis among health care workers. Emerging infectious diseases 17, 488-494 (2011).

2. Menzies, D., Fanning, A., Yuan, L. \& Fitzgerald, M. Tuberculosis among health care workers. The New England journal of medicine 332, 92-98 (1995).

3. Park, J. S. The Prevalence and Risk Factors of Latent Tuberculosis Infection among Health Care Workers Working in a Tertiary Hospital in South Korea. Tuberculosis and respiratory diseases 81, 274-280 (2018).

4. Menzies, D., Joshi, R. \& Pai, M. Risk of tuberculosis infection and disease associated with work in health care settings. The international journal of tuberculosis and lung disease: the official journal of the International Union against Tuberculosis and Lung Disease 11, 593-605 (2007).

5. Nasreen, S., Shokoohi, M. \& Malvankar-Mehta, M. S. Prevalence of Latent Tuberculosis among Health Care Workers in High Burden Countries: A Systematic Review and Meta-Analysis. PloS one 11, e0164034 (2016).

6. Cho, K. et al. Prevalence of latent tuberculosis infection at congregated settings in the Republic of Korea, 2017. J Public Health Wkly Rep 11, 348-354 (2018).

7. Kim, S., Choi, H., Jang, Y. J., Park, S. H. \& Lee, H. Prevalence of and factors related to latent tuberculous infection among all employees in a referral hospital. The international journal of tuberculosis and lung disease: the official journal of the International Union against Tuberculosis and Lung Disease 22, 1329-1335 (2018).

8. Lee, H. et al. Factors associated with non-initiation of latent tuberculosis treatment among healthcare workers with a positive interferon-gamma releasing assay. Scientific reports $\mathbf{9 ,} 61$ (2019).

9. Menzies, D. et al. Adverse events with 4 months of rifampin therapy or 9 months of isoniazid therapy for latent tuberculosis infection: a randomized trial. Annals of internal medicine 149, 689-697 (2008).

10. Goswami, N. D. et al. Predictors of latent tuberculosis treatment initiation and completion at a U.S. public health clinic: a prospective cohort study. BMC public health 12, 468 (2012).

11. Li, J., Munsiff, S. S., Tarantino, T. \& Dorsinville, M. Adherence to treatment of latent tuberculosis infection in a clinical population in New York City. International journal of infectious diseases: IJID: official publication of the International Society for Infectious Diseases 14, e292-297 (2010).

12. Codecasa, L. R. et al. Isoniazid preventive treatment: predictors of adverse events and treatment completion. The international journal of tuberculosis and lung disease: the official journal of the International Union against Tuberculosis and Lung Disease 17, 903-908 (2013).

13. Menzies, D. et al. Four Months of Rifampin or Nine Months of Isoniazid for Latent Tuberculosis in Adults. The New England journal of medicine 379, 440-453 (2018).

14. Pettit, A. C., Bethel, J., Hirsch-Moverman, Y., Colson, P. W. \& Sterling, T. R. Female sex and discontinuation of isoniazid due to adverse effects during the treatment of latent tuberculosis. The Journal of infection 67, 424-432 (2013).

15. Trajman, A. et al. Factors associated with treatment adherence in a randomised trial of latent tuberculosis infection treatment. The international journal of tuberculosis and lung disease: the official journal of the International Union against Tuberculosis and Lung Disease 14, 551-559 (2010).

16. Lee, E. H. et al. Treatment of latent tuberculous infection among health care workers at a tertiary hospital in Korea. The international journal of tuberculosis and lung disease: the official journal of the International Union against Tuberculosis and Lung Disease 22, 1336-1343 (2018).

17. Balkhy, H. H. et al. Compliance with postexposure screening and treatment of latent tuberculosis infection among healthcare workers in a tertiary care hospital in Saudi Arabia. Infection control and hospital epidemiology 35, 176-181 (2014).

18. Shukla, S. J., Warren, D. K., Woeltje, K. F., Gruber, C. A. \& Fraser, V. J. Factors associated with the treatment of latent tuberculosis infection among health-care workers at a midwestern teaching hospital. Chest 122, 1609-1614 (2002).

19. Arguello Perez, E., Seo, S. K., Schneider, W. J., Eisenstein, C. \& Brown, A. E. Management of Latent Tuberculosis Infection Among Healthcare Workers: 10-Year Experience at a Single Center. Clin Infect Dis 65, 2105-2111 (2017).

20. WHO. Latent tuberculosis infection: updated and consolidated guidelines for programmatic management. World Health Organization (2018).

21. Targeted tuberculin testing and treatment of latent tuberculosis infection. This official statement of the American Thoracic Society was adopted by the ATS Board of Directors, July 1999. This is a Joint Statement of the American Thoracic Society (ATS) and the Centers for Disease Control and Prevention (CDC). This statement was endorsed by the Council of the Infectious Diseases Society of America. (IDSA), September 1999, and the sections of this statement. American journal of respiratory and critical care medicine 161, S221-247 (2000).

22. Joint Committee for the Revision of Korean Guidelines for Tuberculosis, Korean Centers for Disease Control and Prevention. Korean guidelines for tuberculosis. Third edition. (2017).

23. Saukkonen, J. J. et al. An official ATS statement: hepatotoxicity of antituberculosis therapy. 174, 935-952 (2006).

\section{Acknowledgements}

This research was supported by Basic Science Research Program through the National Research Foundation of Korea (NRF) funded by the Ministry of Education (NRF-2017R1D1A1B03035267) and a grant of the Korea Health Technology R\&D Project through the Korea Health Industry Development Institute (KHIDI), funded by the Ministry of Health \& Welfare, Republic of Korea (HI15C1543).

\section{Author contributions}

Conception and design: S.J.C., H.L., S-H.K.; Data collection: S.J.C., H.L., G.W.K., J.-H.M., Y.Y., D.W.P., T.S.P., J.-Y. M., T.H.K., J.W.S., H.J.Y., S.-H.K.; Data analysis: S.J.C, H.L., S-H.K., Data interpretation and manuscript writing: S.J.C., H.L., S.-H.K.; Revision of the manuscript and contribution to intellectual content: All authors; S.-H.K. is the guarantor of the manuscript.

\section{Competing interests}

The authors declare no competing interests.

\section{Additional information}

Correspondence and requests for materials should be addressed to S.-H.K.

Reprints and permissions information is available at www.nature.com/reprints. 
Publisher's note Springer Nature remains neutral with regard to jurisdictional claims in published maps and institutional affiliations.

(c) (i) Open Access This article is licensed under a Creative Commons Attribution 4.0 International License, which permits use, sharing, adaptation, distribution and reproduction in any medium or format, as long as you give appropriate credit to the original author(s) and the source, provide a link to the Creative Commons license, and indicate if changes were made. The images or other third party material in this article are included in the article's Creative Commons license, unless indicated otherwise in a credit line to the material. If material is not included in the article's Creative Commons license and your intended use is not permitted by statutory regulation or exceeds the permitted use, you will need to obtain permission directly from the copyright holder. To view a copy of this license, visit http://creativecommons.org/licenses/by/4.0/.

(c) The Author(s) 2020 